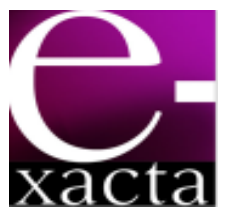

ISSN: 1984-3151

\title{
TEÓFILO OTONI E SUA REGIÃO: PROJETO TOR
}

\section{TEÓFILO OTONI AND ITS REGION: PROJECT TOR}

\section{Leônidas Conceição Barroso}

\author{
Doutor em Ciências em Informática. PUC Rio, 1988 \\ Professor do Programa de Pós-Graduação em Geografia- \\ Tratamento da Informação Espacial da PUC Minas. Belo \\ Horizonte, MG. lbarroso@pucminas.br.
}

Recebido em: 28/08/2012 - Aprovado em: 30/10/2012 - Disponibilizado em: 30/11/2012

RESUMO: TOR é sigla de Teófilo Otoni e sua Região. Este projeto, concebido em 2005, e em execução no Programa de Pós-Graduação em Geografia -Tratamento da Informação Espacial da PUC Minas, tem desenvolvido estudos visando definir os contornos da região da cidade de Teófilo Otoni, vértice da pirâmide da rede urbana do Vale do Mucuri, no extremo nordeste do Estado de Minas Gerais .Nesta apresentação são listados os principais resultados obtidos.

PalAVRAS-ChAVE: Teófilo Otoni. Cidade Média. Vale do Mucuri.

ABSTRACT: TOR is an acronym for Teófilo Otoni and its Region. This project, conceived in 2005, and running in the Graduate Program in Geography of PUC Minas, has developed studies to define the contours of the region leading by Teófilo Otoni, main city of the urban network of Mucuri Valley, in the northeast of the state of Minas Gerais. This paper presents the main results obtained.

KEYWORDS: Teófilo Otoni. Medium size City. Mucuri Valley.

\section{INTRODUÇÃO}

TOR é sigla de Teófilo Otoni e sua Região. Este projeto, concebido em 2005, e em execução no Programa de Pós-Graduação em GeografiaTratamento da Informação Espacial da PUC Minas desde 2006, tem desenvolvido estudos visando definir os contornos da região da cidade de Teófilo Otoni, vértice da pirâmide da rede urbana do Vale do Mucuri, no extremo nordeste do Estado de Minas Gerais.

Os estudos contemplam Teófilo Otoni como cidade média a partir de dois enfoques interdependentes e complementares: o das relações externas da cidade e o de sua estrutura interna (em comparação com as demais cidades médias da região e com os modelos morfológicos das cidades médias de Minas Gerais).

Além disso, tem sido elaboradas comparações sobre a evolução geoeconômica da região a partir de 1970, produzindo-se mapas e textos explicativos sobre os vários aspectos analisados na busca da caracterização da região de Teófilo Otoni. A FIG.1 mostra uma vista parcial do centro da cidade. 


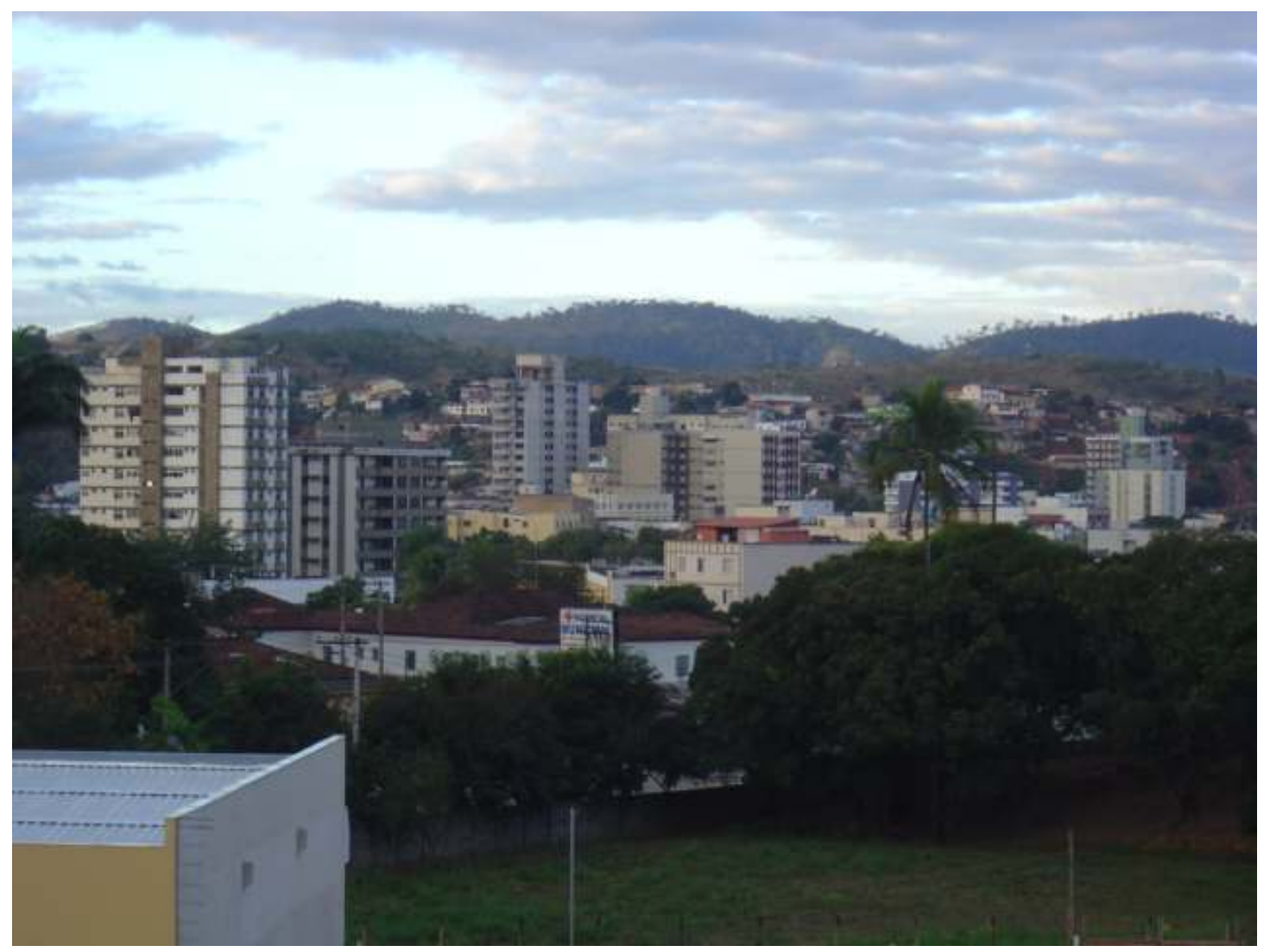

Figura 1 - Vista parcial do centro de Teófilo Otoni

Foto de João Luís de Araújo Barroso, 2010.

O projeto tem sido desenvolvido por meio de tratamentos quantitativos, gráficos e cartográficos das informações contidas nos bancos de dados dos Laboratórios do Programa de Pós-Graduação em Geografia-Tratamento da Informação Espacial da PUC Minas com relação aos aspectos: ambientais, urbanos e regionais. É utilizado o ambiente SIG - Sistema de Informações Geográficas - para a elaboração de mapas temáticos a partir dos resultados obtidos.

Os principais equipamentos secundários e terciários, responsáveis pela "polarização" da cidade de Teófilo Otoni também tem sido objeto de investigação.

De grande importância são os trabalhos de campo realizados periodicamente em Teófilo Otoni e região para atualização dos bancos de dados do referido Programa de Pós-graduação.

\section{Resultados de Dissertações e de Teses}

A primeira fase do Projeto TOR (2006-2011) gerou novas hipóteses para o prosseguimento de estudos sobre Teófilo Otoni e sua região, evidenciando a compreensão das características dos diferenciais sócio-econômicos, sócio-ambientais e infraestruturais.

Titularam-se novos mestres e doutores no Programa de Pós-Graduação em Geografia - Tratamento da Informação Espacial, com conhecimentos aprofundados sobre Teófilo Otoni e região permitindo a produção de bibliografia científica com a publicação de artigos para revistas científicas e anais de Congressos Nacionais e Internacionais. Os primeiros resultados foram obtidos já em 2006. 
Christiano Ottoni Carvalho defendeu sua dissertação de mestrado: "A mesorregião do Vale do Mucuri: Contextualização Histórica e Análise do Desenvolvimento", orientada por Leônidas Conceição Barroso e coorientada por Oswaldo Bueno Amorim Filho e João Francisco de Abreu.

Carvalho (2006) tratou a contextualização histórica e análise do desenvolvimento da Mesorregião expandida do Vale do Jequitinhonha e Mucuri, composta por 105 municípios, com a intenção de identificar descontinuidades temporais e espaciais nesta região, que nasce de maneira integrada e se desarticula ao longo do tempo por diversos fatores, tanto políticos, quanto sócio-econômicos. A abordagem foi feita por meio da análise do mapeamento dos Índices de Desenvolvimento Humano - IDH's da Mesorregião no período compreendido entre 1991 e 2000, utilizando de técnicas de SIG e análise de superfícies geoestatísticas.

Em 2007 Cynthia Andréia Antão Pires defendeu a dissertação de mestrado intitulada "Estratégia de Saúde da Família na cidade de Teófilo Otoni - MG: Perspectivas Geográficas de uma rede de saúde no espaço intra-urbano", orientada por Leônidas Conceição Barroso e coorientada por Alexandre Magno Alves Diniz.

Pires (2007) analisa a rede básica de serviços de saúde de Teófilo Otoni - MG, com enfoque na organização espacial da Estratégia de Saúde da Família e suas relações espaciais com os territórios da área urbana do município.

Além de mapear as áreas de abrangência dos Postos do Programa de Saúde da Família, a autora elabora diagnóstico sócio-econômico da cidade de Teófilo Otoni, valendo-se de variáveis construídas a partir de dados do Censo Demográfico de 2000. Tais variáveis referem-se às dimensões de renda, escolaridade, características sociais e saneamento dos domicílios particulares permanentes e seus respectivos residentes, para cada um dos 96 setores censitários da área urbana. Setor Censitário é a unidade territorial mínima criada para fins de controle cadastral da coleta de dados do Censo Demográfico.

A análise das variáveis foi feita com a utilização da técnica de Análise de Componentes Principais, permitindo a atribuição de pesos às variáveis, que captaram o maior percentual da variabilidade máxima dos dados. O mapeamento da vulnerabilidade sócioeconômica foi construído com base nos valores obtidos na primeira componente para os 96 setores censitários da cidade. Por meio desta técnica foi possível identificar os diferenciais internos de vulnerabilidade sócio-econômica dos moradores da cidade. A dissertação gerou o trabalho Estratégia de Saúde da Família na cidade de Teófilo Otoni - MG: Diagnósticos e Perspectivas. (PIRES, 2009).

Flávio Apolinário produz em 2010 a dissertação de mestrado: "A rede Urbana da Mesorregião do Vale do Mucuri: uma proposta de hierarquização por meio de técnicas de estatística multivariada", orientada por Leônidas Conceição Barroso e coorientada por Alexandre Magno Alves Diniz.

Apolinário (2010) faz uma análise de como se encontra estruturada a rede de cidades da mesorregião do Vale do Mucuri, no Estado de Minas Gerais e propõe uma hierarquização das cidades por meio do estudo e interpretação dos dados referentes à oferta de serviços e infraestrutura urbana, coletados para cada um dos vinte e três municípios que compõem a mesorregião.

Para investigar a organização da rede urbana da mesorregião do Vale do Mucuri foram utilizadas técnicas de estatística multivariada (Análise de Agrupamentos e Análise de Componentes Principais), que auxiliaram na geração de um índice de mensuração do grau de acessibilidade aos serviços urbanos oferecidos por cada cidade da região 
estudada. A este índice chamou-se de IAU (Índice de Acessibilidade Urbana).

Por meio do índice de acessibilidade urbana, Apolinário propôs uma hierarquização da rede urbana do Vale do Mucuri.

Alan Nunes Araújo produz em 2011 a dissertação de mestrado: "Mesorregião do Vale do Mucuri - MG: a influência dos eixos viários na evolução de sua rede urbana" orientada por Leônidas Conceição Barroso e coorientada por Oswaldo Bueno Amorim Filho.

Araújo (2011a) procura salientar o papel dos eixos de comunicação viária no desbravamento e posterior desenvolvimento urbano e regional do Vale do Mucuri, região de ocupação tardia da Província de Minas Gerais.

Com o declínio das atividades mineradoras, a Comarca de Serro Frio volta-se à agricultura e à criação de gado, gerando a necessidade de barateamento de fretes, além da redução de tempo de viagem para o escoamento da crescente produção. O Vale do Mucuri torna-se atrativo por possibilitar uma ligação por terra, mais curta, com o litoral: a porção sul da província da Bahia e a porção noroeste da província do Espírito Santo.

O autor, partindo da hipótese de que os eixos viários são fatores que tem influenciado a distribuição espacial ao longo da história de ocupação, utiliza mapas e cartas dos séculos XIX e XX com a finalidade de acompanhar o surgimento e a evolução dos eixos que existiram no Vale do Mucuri, utilizando novas propostas metodológicas.

Este estudo tem como suporte as tecnologias de Sistemas de Informações Geográficas que fornecem recursos para o tratamento destes documentos, possibilitando ligação efetiva entre o passado e o presente. (ARAÚJO, 2011b)

Margareth Alves Carvalho, em 2011, produz sua tese de doutorado "O espaço intra-urbano de Teófilo
Otoni/MG”, orientada por Leônidas Conceição Barroso e coorientada por Oswaldo Bueno Amorim Filho.

Carvalho (2011) analisa o espaço intraurbano de Teófilo Otoni e a sua configuração em relação aos novos eixos de crescimento da cidade. A análise vem somar esforços aos estudos referentes às cidades médias, que vêm se constituindo em elementos fundamentais nos processos de desenvolvimento regional e urbano.

Além do reconhecimento enquanto áreas de atração regional, as cidades médias assumem um importante papel na interligação entre as redes locais e globais. Esse é o caso da cidade de Teófilo Otoni, localizada na região de planejamento do Jequitinhonha/Mucurinordeste do estado de Minas Gerais - região essa que se encontrava em processo de estagnação econômica desde a década de 80 e que vive agora um novo ciclo de desenvolvimento.

\section{Seminário Teófilo Otoni e Bacia do MucURI: Diagnósticos e PeRsPectivas}

Em comemoração ao bicentenário de nascimento de Theophilo Benedicto Ottoni, em 2007, foi realizado pelo Programa de Pós Graduação em Geografia Tratamento da Informação Espacial com o apoio da Prefeitura Municipal de Teófilo Otoni, por meio de sua Secretaria de Educação e Diretoria de Cultura, na cidade de Teófilo Otoni, o SEMINÁRIO "Teófilo Otoni e Bacia do Mucuri: Diagnósticos e Perspectivas”, sob a coordenação de Leônidas Conceição Barroso e com a contribuição de vários professores e alunos.

Estiveram presentes no SEMINÁRIO os professores: doutores Oswaldo Bueno Amorim Filho, Ruibran Januário dos Reis, Márcia Maria Duarte dos Santos e Magali Maria de Araújo Barroso, convidada do UniBH. E os alunos Alan Nunes Araújo, Ana Paula Teixeira, Cláudia Cristina Rios Caxias da Costa, Cynthia Andréia Antão Pires e Wagner Barbosa Batella. 
Dentre as principais contribuições do evento destacam-se os artigos de Oswaldo Bueno Amorim Filho: Teófilo Otoni em seu ambiente natural e Teófilo Otoni em suas redes urbanas.

No Apêndice encontra-se a programação do evento com as palestras apresentadas pelos diversos participantes.

\section{OUtros Trabalhos}

2010: "Breve resgate da Geografia Histórica de Teófilo Otoni”. Autores: Leônidas Conceição Barroso, Margareth Alves Carvalho e Alan Nunes Araújo. (BARROSO; CARVALHO; ARAÚJO, 2010).

2011: Fatores determinantes na conformação atual do Vale do Mucuri. Autores: Alan Nunes Araújo e Leônidas Conceição Barroso. IV Simpósio Luso Brasileiro de Cartografia Histórica. Porto, Portugal

\section{EquiPe de Pesquisadores}

\section{Professores do Programa}

Prof. Dr. Leônidas Conceição Barroso

Prof. Dr. Oswaldo Bueno Amorim Filho

Prof. Dr. João Francisco de Abreu

Prof. Dr. Alexandre Magno Alves Diniz

\section{Professoras Convidadas}

Prof. ${ }^{a}$ Dr. ${ }^{a}$ Márcia Maria Duarte dos Santos (UFMG)

Prof. ${ }^{a}$ Dr. ${ }^{a}$ Magali Maria de Araújo Barroso (UniBH)

\section{Aluno(a)s do Programa de doutorado \\ Cláudia Cristina Caxias Rios da Costa \\ Margareth Alves Carvalho}

\section{Aluno(a)s do Programa de mestrado}

Alan Nunes Araújo

Christiano Ottoni de Carvalho

Cynthia Andreia Antão Pires

Flávio Apolinário

\section{AgRAdeCIMENTOS}

Várias pessoas e instituições colaboraram para o sucesso do projeto TOR. O autor agradece às Instituições:

Em Belo Horizonte:

- PUC Minas por meio de seu Programa de Pós-Graduação em Geografia-Tratamento da Informação Espacial.

- $\quad$ AFATO

Em Teófilo Otoni:

- Prefeitura Municipal de Teófilo Otoni,

- Instituto Pró Rio Todos os Santos e Mucuri,

- Agência de Desenvolvimento do Mucuri,

- Associação dos Municípios do Mucuri-AMUC,

- IESFATO

Na PUC Minas, professores, alunos e funcionários não mediram esforços para que o projeto obtivesse sucesso.

Agradecimentos especiais ao professor Dr. Oswaldo Bueno Amorim Filho, consultor do projeto, autor do modelo morfológico funcional para as cidades médias e grande incentivador do Projeto TOR. Sua presença no projeto muito contribuiu para a obtenção de resultados significativos.

Aos professores Dr. Alexandre Magno Alves Diniz e Dr. João Francisco de Abreu, coorientadores de dissertações de mestrado. Ao professor Dr. Ruibran 
Januário dos Reis que esteve presente no Seminário em Teófilo Otoni.

Aos alunos de mestrado e doutorado do Programa cujas dissertações e teses tiveram como objeto de pesquisa o município de Teófilo Otoni e o Vale do Mucuri.

Fora da PUC Minas, à professora $\mathrm{Dr}^{\text {a }}$ Márcia Maria Duarte dos Santos, do Centro de Referência em Cartografia Histórica da UFMG que esteve presente no Seminário em Teófilo Otoni enquanto era professora visitante do PPGG-TIE.

À professora Dr. ${ }^{a}$ Magali Maria de Araújo Barroso, que também participou do Seminário em Teófilo Otoni e hoje é editora geral da Revista e-xacta do UniBH.

Ao Gecernir Colen, presidente da Associação dos Filhos e Amigos de Teófilo Otoni - AFATO e ao jornalista Arnaldo Gomes Pinto Júnior, diretor de comunicação da AFATO.

Em Teófilo Otoni o Projeto TOR contou com o prestimoso apoio de várias pessoas e entidades.

O autor agradece à professora Maria José Haueisen Freire, prefeita municipal (2005-2012) de Teófilo Otoni no período em que o Projeto TOR foi desenvolvido e cujo apoio foi decisivo. À lldete José da Silva Mota, chefe de gabinete da Prefeita. Às professoras Maria Helena Costa Salim, Secretária Municipal de
Educação e Beatriz Marques Faria, Diretora de Cultura que apoiaram o projeto, principalmente para a realização do Simpósio Bacia do Mucuri: diagnósticos e perspectivas, realizado em Teófilo Otoni em 22 de agosto de 2007, por ocasião do bicentenário de nascimento Teófilo Benedito Otoni. Ao secretário de saúde Gilberto Leonhardt

À ambientalista Alice Lorentz Faria Godinho, presidente do Instituto Pró Rios Todos os Santos e Mucuri, companheira de ideais sempre em defesa do Vale do Mucuri. Ao empresário lesser Aniz Lauar, presidente da Agência de Desenvolvimento do Mucuri, grande incentivador de estudos e ações em favor de Teófilo Otoni e região.

A Iris Soriano Miglio e Gilberto Ottoni Porto, do Instituto Histórico e Geográfico do Mucuri, ao Padre Joel Ferreira da Silva, diretor do IESFATO pelo auxilio prestado nos trabalhos de campo. Ao historiador professor Márcio Achtschin, companheiro de trabalho de campo ao Alto dos Bois.

Ao Igor Sorel Tavares, companheiro de primeiras horas, grande conhecedor do Vale do Mucuri, sempre com disposição e boa vontade nos trabalhos de campo na Estrada Santa Clara e no Alto dos Bois. A Minie (Maria de Fátima) Martins, que com competência e alegria documentou fotograficamente os trabalhos de campo.

\section{REFERÊNCIAS}

APOLINÁRIO, F. A Rede Urbana da Mesorregião do Vale do Mucuri. Dissertação (Mestrado em Geografia - Tratamento da Informação Espacial)-Pontifícia Universidade Católica de Minas Gerais. Belo Horizonte, 2010. Disponível em: $<$ http://www.biblioteca.pucminas.br/teses/TratInfEspaci al ApolinarioF 1.pdf>.

ARAÚJO, A. N; A Influência dos Eixos Viários na Rede Urbana do Mucuri-MG. Dissertação (Mestrado em Geografia - Tratamento da Informação Espacial) Pontifícia Universidade Católica de Minas Gerais. Belo Horizonte, 2011a.
ARAÚJO, A. N; Fatores Determinantes na Conformação do Vale do Mucuri-MG .In: IV SIMPÓSIO LUSO BRASILEIRO DE CARTOGRAFIA HISTÓRICA, 2011b. Porto, Portugal. Anais Eletrônicos... Vol. Único. p. 1-14. Disponível em: http://eventos.letras.up.pt/ivslbch/comunicacoes/93.pdf Acesso em: 10 ago.2012.

BARROSO, L. C.; CARVALHO, M.A.; ARAÚJO, A.N; Breve resgate da Geografia Histórica de Teófilo Otoni. In: GODINHO, A. L. F. (Org.) Expedição Mucuri. Belo Horizonte: Movimento Pró Rio Todos os Santos e Mucuri, 2010, p. 27-42. 
CARVALHO, C. O. A Contextualização Histórica e Análise do Desenvolvimento da Mesorregião do Vale do Mucuri. 2006. Dissertação (Mestrado em Geografia - Tratamento da Informação Espacial)Pontifícia Universidade Católica de Minas Gerais. Belo Horizonte, 2006.

CARVALHO, M. A. O Espaço Intraurbano da cidade de Teófilo Otoni-MG. Tese (Doutorado em Geografia - Tratamento da Informação Espacial)-Pontifícia Universidade Católica de Minas Gerais. Belo Horizonte, 2011.
PIRES, C. A. A.; Estratégia de Saúde da Família na cidade de Teófilo Otoni-MG: Perspectivas Geográficas no espaço intraurbano. Dissertação (Mestrado em Geografia - Tratamento da Informação Espacial)-Pontifícia Universidade Católica de Minas Gerais. Belo Horizonte, 2007. Disponível em: http://www.biblioteca.pucminas.br/teses/TratInfEspacia I PiresCA 1.pdf>. Acesso em: 20 ago. 2012.

PIRES, C. A. A. Estratégia de Saúde da Família na cidade de Teófilo Otoni-MG: Diagnósticos e Perspectivas. Caderno de Geografia. v. 19. p. 11-27. 2009. 
Programa de Pós-Graduação em Geografia Tratamento da Informação Espacial

\section{Seminário}

Teófilo Otoni e Bacia do Mucuri:

Diagnósticos e Perspectivas

Bicentenário de nascimento de Theophilo Benedicto Ottoni Sesquicentenário da Estrada Santa Clara - Filadélfia

Teófilo Otoni - MG

22 agosto de 2007 


\section{APRESENTAÇÃO}

O Programa de Pós-Graduação em Geografia - Tratamento da Informação Espacial da Pontifícia Universidade Católica de Minas Gerais desenvolve, desde março de 2005, o Projeto TOR - Teófilo Otoni e sua Região, cujas pesquisas contemplam a cidade de Teófilo Otoni e a região do Vale do Mucuri. Este programa acadêmico busca interagir com a realidade da bacia do Mucuri e de sua cidade primaz.

Vários trabalhos já foram concluídos, dentre os quais duas dissertações de mestrado. Vale ressaltar que pesquisas em nível de doutorado, mestrado e iniciação científica estão em andamento contemplando aspectos diversos da cidade de Teófilo Otoni e do Vale do Mucuri. As pesquisas são financiadas por projetos de docentes com recursos da CAPES, do CNPq, da FAPEMIG e do FIP/PUC Minas. Além disso, o Programa conta com o apoio da Prefeitura Municipal de Teófilo Otoni para os trabalhos de campo no município.

Neste seminário procura-se compartilhar com gestores públicos e privados, acadêmicos e outros segmentos da sociedade os resultados das pesquisas em andamento e das já concluídas. Com isso, busca-se estimular parcerias, que possam colaborar na proposição de planos de ação para o encaminhamento de possíveis soluções de problemas no âmbito do Vale do Mucuri.

O evento contempla os seguintes temas:

Redes urbanas

Climatologia

Localização

Demografia

Geografia da Saúde

Geografia do Crime

Geografia Histórica

Cartografia

Nós, do Programa de Pós-graduação em Geografia - Tratamento da Informação Espacial da Pontifícia Universidade Católica de Minas Gerais, sentimo-nos honrados em participar deste momento histórico de Teófilo Otoni e do Vale do Mucuri, em que são comemorados o 200. ${ }^{\circ}$ Aniversário de nascimento de Theophilo Benedicto Ottoni e o $150 .^{\circ}$ Aniversário da Estrada Santa Clara - Fladélfia.

$\mathrm{Na}$ impossibilidade de agradecer individualmente a cada um, deixamos aqui nossos agradecimentos a todos aqueles que colaboraram para tornar possível a realização deste encontro, tanto no nosso Programa em Belo Horizonte quanto na Prefeitura de Teófilo Otoni.

Teófilo Otoni, 22 de agosto de 2007.

Prof. Dr. Leônidas Conceição Barroso Coordenador do Seminário Teófilo Otoni e Bacia do Mucuri: Diagnósticos e Perspectivas 


\section{SEMINÁRIO}

\section{Teófilo Otoni e Bacia do Mucuri: Diagnósticos e Perspectivas}

\section{REALIZAÇÃO}

Programa de Pós-graduação em Geografia - Tratamento da Informação Espacial da PUC Minas

Prefeitura Municipal de Teófilo Otoni

\section{LOCAL}

Teófilo Otoni, Minas Gerais

Auditório do SESC

\section{DATA}

22 de agosto de 2007

\section{PROGRAMA}

\section{8:00 CREDENCIAMENTO}

08:30 Abertura oficial

Sra. Maria José Haueisen Freire - Prefeita Municipal de Teófilo Otoni

08:45 A Rede Urbana do Vale do Mucuri

Prof. Dr. Oswaldo Bueno Amorim Filho (PUC Minas)

\section{0:15 INTERVALO}

10:30 Climatologia do Vale do Mucuri

Prof. Dr. Ruibran Januário dos Reis (PUC Minas)

11:15 Localização de serviços: perspectivas de aplicabilidade

Profa. Dra. Magali Maria de Araújo Barroso (UniBH, Pesquisadora Associada da PUC Minas)

\section{2:00 ALMOÇO}

14:00 Estratégias do Programa de Saúde da Família da cidade de Teófilo Otoni

Cynthia Andréia Antão Pires, Mestre (PPG-TIE/PUCMinas)

14:40 Fluxos migratórios dos municípios da microrregião de Teófilo Otoni - 1986-1991 e 1995-2000

Cláudia Cristina Rios Caxias da Costa, Doutoranda (PPG-TIE/PUC Minas)

15:20 Criminalidade violenta no Vale do Mucuri: Contribuições da Geografia do Crime.

Wagner Barbosa Batella, Mestrando (PPG-TIE/PUCMinas)

\section{6:00 INTERVALO}

16:20 Cartografia e identidade geográfica, histórica e cultural do Vale do Mucuri

Profa. Dra. Márcia Maria Duarte dos Santos (PUC Minas)

17:00 Mapa digital interativo da Estrada Santa Clara - Filadélfia

Alan Nunes Araújo - Graduando, (Geografia/PUC Minas em Contagem)

\section{ENCERRAMENTO}

17:30 Profa. Maria Helena Costa Salim - Secretária Municipal de Educação

SAGUÃO

10:00 às 18 horas: SESSÃO DE PÕSTERES 


\section{SEMINÁRIO}

\section{TEÓFILO OTONI E BACIA DO MUCURI: Diagnósticos e Perspectivas \\ Auditório do SESC - Teófilo Otoni, MG \\ 22 de agosto de 2007}

\section{Comissão de honra}

Profa. Maria José Haueisen Freire

Prefeita Municipal de Teófilo Otoni

Prof. Dr. João Francisco de Abreu

Pró-Reitor de Pesquisa e Pós-graduação da PUC Minas

\section{Comissão Organizadora (Belo Horizonte)}

Prof. Dr. Leônidas Conceição Barroso - PUC Minas

Coordenador do Seminário

Prof. Dr. Oswaldo Bueno Amorim Filho

Coordenador do Programa de Pós-graduação em Geografia-

Tratamento da Informação Espacial - PUC Minas

Prof. Dr. José Flávio Morais Castro

Coordenador Adjunto do Programa de Pós-graduação em Geografia-

Tratamento da Informação Espacial - PUC Minas

\section{Comissão Organizadora (Teófilo Otoni)}

Profa. Maria Helena Costa Salim

Secretária Municipal de Educação de Teófilo Otoni

Profa. Beatriz Farias Marques

Diretora de Cultura de Teófilo Otoni

Profa. Íris Soriano

Presidente do Instituto Histórico e Geográfico do Vale do Mucuri 